\title{
Performance Benefits of Self-Assembly in a Swarm-Bot
}

\author{
Rehan O'Grady $^{1}$, Roderich Groß ${ }^{1,2}$, Anders Lyhne Christensen ${ }^{1}$, \\ Francesco Mondada ${ }^{3}$, Michael Bonani ${ }^{3}$ and Marco Dorigo ${ }^{1}$ \\ ${ }^{1}$ IRIDIA-CoDE, Université Libre de Bruxelles, Brussels, Belgium \\ ${ }^{2}$ Unilever R\&D Port Sunlight, Bebington, United Kingdom \\ ${ }^{3}$ LSRO, Ecole Polytechnique Fédérale de Lausanne, Switzerland \\ Email: \{rogrady,rgross,alyhne,mdorigo\}@ulb.ac.be, \{francesco.mondada,michael.bonani\}@epfl.ch
}

\begin{abstract}
Mobile robots are said to be capable of selfassembly when they can autonomously form physical connections with each other. Despite the recent proliferation of selfassembling systems, little work has been done on using selfassembly to add functional value to a robotic system, and even less on quantifying the contribution of self-assembly to system performance.

In this study we demonstrate and quantify the performance benefits of i) acting as a physically larger self-assembled entity, ii) using self-assembly adaptively and iii) making the robots morphologically aware (the self-assembled robots leverage their new connected morphology in a task specific way).

In our experiments, two real robots must navigate to a target over a-priori unknown terrain. In some cases the terrain can only be overcome by a self-assembled connected entity. In other cases, the robots can reach the target faster by navigating individually.
\end{abstract}

\section{INTRODUCTION}

Self-assembly is a widely observed naturally occurring phenomenon [16]. Self-assembly adds functional value to biological systems and has evolved both at the cellular level and at the level of distinct organisms [1], [2]. Members of the ant species Ecophylla longinoda, for example, link to one another to form bridges that other ants can then traverse [9].

Self-assembly in biological systems has been of inspiration to the robotics community [5]. This study concerns the use of self-assembly with autonomous robots [7]. Existing systems of particular relevance, therefore, are those in which the components that assemble are self-propelled. A variety of such systems have been implemented and studied over the past fifty years [4], [6], [8], [12], [14], [17], [18]. However, there has been little research on how self-assembly can add functional value to a system of autonomous robots, and even less on quantifying the contribution of self-assembly to system performance.

In this study we consider a task that requires two real robots to navigate towards a target light source over a-priori unknown terrain. In some of our experimental environments the terrain that the robots encounter can be navigated by a single robot individually. In other environments, the robots must self-assemble and navigate as a connected entity to successfully reach the target.

We demonstrate and quantify the contribution to system performance of the self-assembly mechanism. Initially, we consider the simple benefits of scale conferred by selfassembly: some tasks can be carried out more effectively if the robots act as a larger connected entity [10]. For example, a larger robotic entity might have increased stability for rough terrain navigation [6] or increased strength for object transportation [15].

We go on to show that the system is more efficient if the robots choose autonomously when and if to assemble based on the environment they encounter. Such adaptivity is innovative, as in most existing studies on self-assembly in collective (and modular) robotics the robots are statically programmed in advance either to act individually or to selfassemble. Typically, the experiment is set up to provide a context in which the pre-programmed behaviour appears meaningful. In this study, we quantify the benefits of adaptive self-assembly over and above static pre-programmed behaviour, extending our previous work in which we implemented an adaptive self-assembly mechanism [13].

Finally, we argue that self-assembling systems can improve their performance by making the connecting robots 'morphology aware'. We present a mechanism through which the self-assembled robots leverage their new connected morphology in a task oriented way. For our task this means that the self-assembled robotic entity rotates its body with respect to the terrain it encounters so as to maximise its stability.

\section{EXPERIMENTAL SETUP}

\section{A. The S-Bot}

We use the SWARM-BOT robotic platform [11]. This platform is made up of independent mobile autonomous robots called s-bots (see Fig. 1) that can form physical connections with each other. The entity formed by two or more connected s-bots is called a swarm-bot.

The $s$-bot is $12 \mathrm{~cm}$ high without its perspex camera turret, and has a diameter of $12 \mathrm{~cm}$ without its gripper. Thanks to its traction system that combines tracks and wheels, the $s$-bot is mobile on uneven terrain whilst still retaining the ability to rotate on the spot efficiently. The main $s$-bot body houses most of its sensory and processing systems and can rotate with respect to the chassis by means of a motorised axis.

Physical connections between $s$-bots are established by a gripper-based connection mechanism. Each s-bot is surrounded by a transparent ring that can be grasped by other 


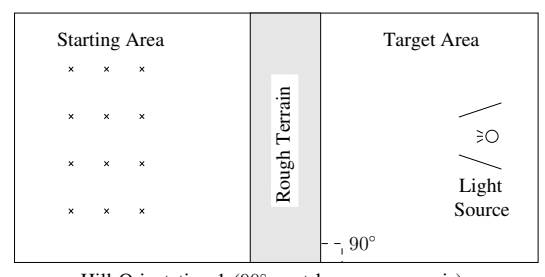

Hill Orientation $1\left(90^{\circ}\right.$ w.r.t longer arena axis)

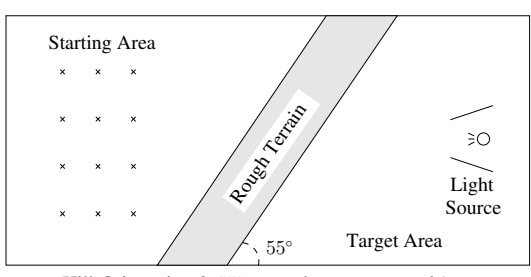

Hill Orientation $2\left(55^{\circ}\right.$ w.r.t. longer arena axis)

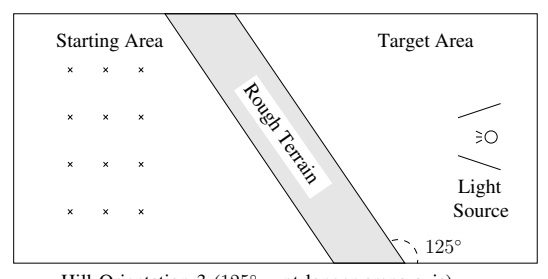

Hill Orientation $3\left(125^{\circ}\right.$ w.r.t longer arena axis)

Fig. 2. Scale diagram of the three possible hill orientations. The arena for each of the seven environments used in this study measures $210 \mathrm{~cm} x \mathrm{x} 105 \mathrm{~cm}$. Three environments contain the 'moderate' hill $(2.8 \mathrm{~cm}$ high, navigable by a single $s$-bot). Three environments contain the 'difficult' hill ( $6.5 \mathrm{~cm}$ high, not navigable by a single $s$-bot). One environment has no hill. The starting area and target area are demarcated by the hill and the arena boundaries (in the no-hill environment the target area is considered to be the same as for environments with hill orientation 1). Starting positions are marked by crosses.

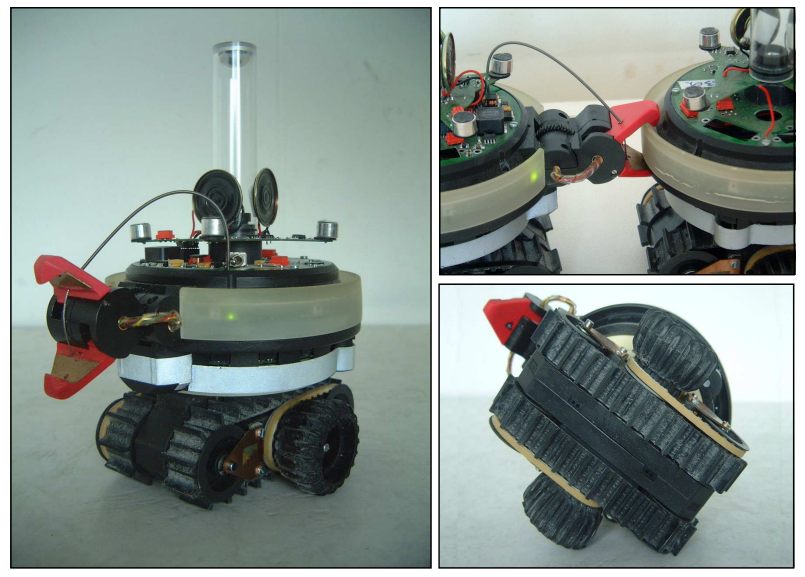

Fig. 1. Left: The s-bot. Above Right: The $s$-bot connection mechanism. Below Right: The s-bot traction system.

$s$-bots. An optical light barrier inside the $s$-bot gripper indicates when another s-bot's ring (or another graspable object) is between the jaws of the gripper. $S$-bots advertise their location by means of 8 sets of RGB coloured LEDs distributed around the inside of their transparent ring. These LEDs can also provide indications of the $s$-bot's internal state to other nearby $s$-bots.

The $s$-bot has an omni-directional camera that depending on light conditions can detect other $s$-bots' LEDs up to $40 \mathrm{~cm}$ away or an external light source up to $200 \mathrm{~cm}$ away. The $s$ bot has 15 proximity sensors distributed around its body that allow for the detection of obstacles. A 3-axes accelerometer provides information on the $s$-bot's inclination that can be used to detect if the $s$-bot is in danger of falling.

Other sensors provide the $s$-bot with proprioceptive information about its internal motors. This includes positional information (e.g., of the rotating turret) and torque information (e.g., of forces acting on the traction system).

\section{B. The Task}

We conduct experiments in seven different environments (see Fig. 2). In each environment we designate a 'starting area' and a 'target area'. Six of the seven environments contain a hill. In these environments the target area is the region containing the light source demarcated by the hill and the walls of the arena. In the hill-less environment we designate the 'target' area as a specific rectangular region containing the light source.

We use two types of hill — a 'moderate' hill and a 'difficult' hill. The moderate hill is $2.8 \mathrm{~cm}$ high and can be overcome by a single $s$-bot. The difficult hill is $6.5 \mathrm{~cm}$ high and is only navigable if two or more $s$-bots assemble into a bigger connected entity (the steepness of the hill would cause a single $s$-bot to topple).

The initial position of each $s$-bot in the starting area is assigned randomly by uniformly sampling without replacement from a set of 12 possible starting points. The $s$-bot's initial orientation is chosen randomly from a set of 4 possible directions. To complete the task the s-bots must reach the target area without toppling over.

\section{Simple Benefits of ScAle}

In this section we consider the simple benefits of scale derived by acting as a larger connected entity. We conducted experiments with two different controllers in the difficult hill environments. When executing the adaptive self-assembly controller, the s-bots make use of self-assembly in order to overcome the hill. When executing the phototaxis only controller, the s-bots attempt to navigate to the target individually. We first present the two controllers, then discuss their relative performance.

\section{A. Control}

All of the controllers presented in this study (not just in this section) have the following features in common: each $s$ bot is completely autonomous, and has no a-priori knowledge of the environment it is in or of its initial position and orientation. A single controller is copied onto each of the $s$-bots and executed on each of the s-bots independently. Communication (when used) is visual and strictly local the $s$-bots illuminate their LED rings with different colours to advertise their location and to provide indications of their internal state to other s-bots within visual range.

1) Adaptive Self-Assembly Controller: The controller is a finite state machine (see Fig. 3). Fig. 4 illustrates a two $s$-bot system executing the controller. An $s$-bot starts by illuminating its blue LEDs and navigating independently towards the target light source (state Solo_Phototaxis). If the $s$-bot finds itself on a hill too difficult for it to pass alone (i.e., if its 3D accelerometers detect an inclination angle that exceeds a preprogrammed threshold), or if it sees a green $s$-bot or a 


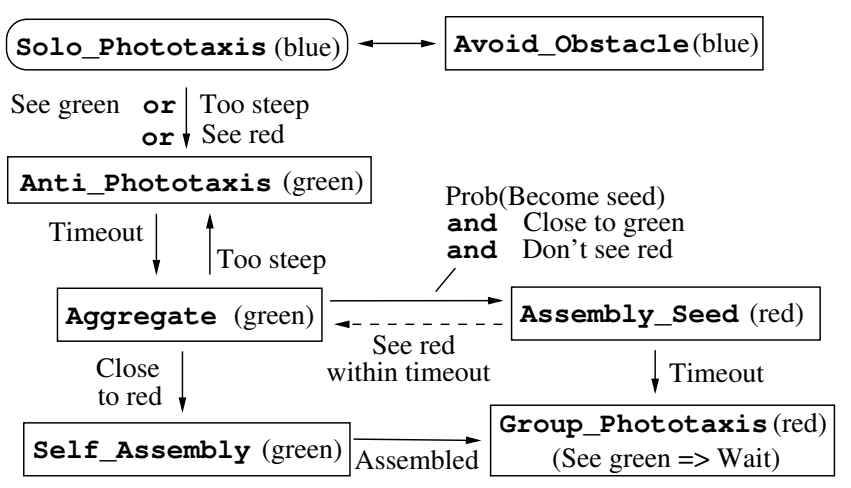

Fig. 3. Finite State Machine representing the Adaptive Self-Assembly Controller. The starting state is Solo_Phototaxis. Colours in parentheses refer to the LEDs that are illuminated in the corresponding state.

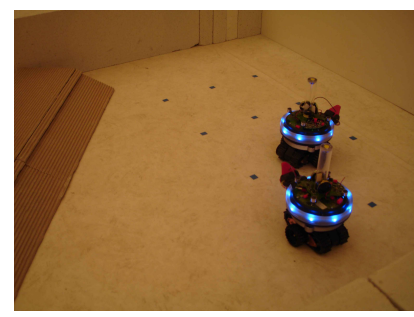

(a)

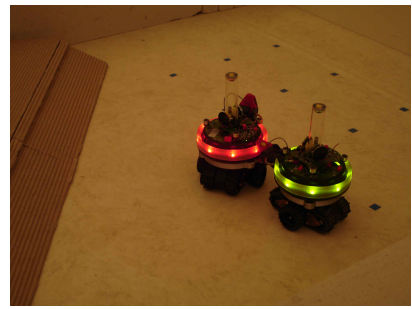

(c)

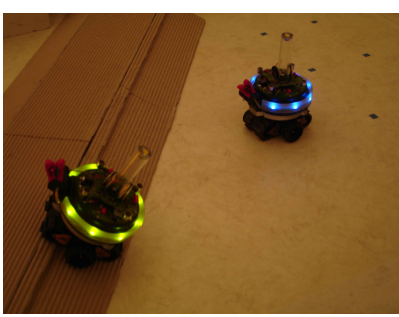

(b)

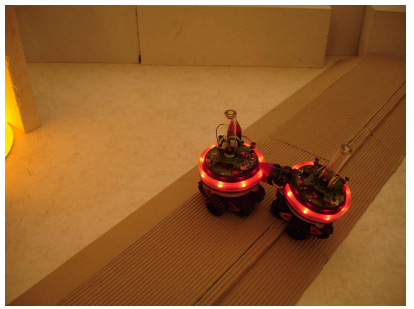

(d)
Fig. 4. (a): Two s-bots start from random positions and orientations. Initially, they perform individual phototaxis, and have their blue LEDs illuminated. (b): One s-bot detects a slope it cannot overcome alone and illuminates green LEDs. The other $s$-bot detects colour green (local communication). (c): The group aggregates and self-assembles. (d): The $s$-bots collectively overcome the rough terrain and reach the target area

red $s$-bot, it retreats away from the hill for a given length of time (state Anti_Phototaxis). It then switches into state Aggregate, illuminates its green LEDs and tries to get close to a red (assembled) $s$-bot, or if no red $s$-bot is perceived, to search for and get close to another green (aggregating) $s$-bot. In the latter case, if the $s$-bot is sufficiently close to another green $s$-bot and can still see no other red $s$ bots, it can trigger self-assembly with a given probability by becoming a stationary seed (state Assembly_Seed). A seed $s$-bot lights up its red LEDs, and waits until a timeout has expired. If it sees another red $s$-bot within the timeout period, it reverts to state Aggregate. Otherwise, after the timeout it switches to state Group_Phototaxis. If an aggregating $s$-bot gets sufficiently close to a red (assembled) $s$-bot, then it starts self-assembling (state Self_Assembly). Assembled $s$-bots switch to state Group_Phototaxis. An s-bot in state Group_Phototaxis illuminates its red LEDs, and navigates (collectively) to the target light source once it can no longer detect any green (aggregating) s-bots. ${ }^{1}$

2) Phototaxis Only Controller: This controller is a modified version of the adaptive self-assembly controller in which the transition to state Anti_Phototaxis is disabled. Thus, only the states Solo_Phototaxis and Avoid_Obstacle are executed. Each s-bot moves independently towards the light at a constant speed irrespective of the type of terrain it encounters.

\section{B. Comparative Performance}

We conducted 60 trials with each controller in the difficult hill environments ( 20 trials per hill orientation). To minimise damage to the robots, we used just a single robot for the trials of the phototaxis-only controller. ${ }^{2}$

When executing the phototaxis-only controller, the s-bot failed to overcome the hill in all 60 trials. In each trial the $s$-bot reached the hill and then toppled backwards due to the steepness of the slope. To confirm that the s-bot was failing due to the intrinsic properties of the slope, we repeated this experiment at a number of different constant speeds and observed the same result.

When executing the adaptive self-assembly controller, both $s$-bots successfully self-assembled into a two s-bot swarmbot in every trial. In 21 trials $(35 \%)$ the swarm-bot succeeded in overcoming the hill, and thus completed the task. In the other 39 trials $(65 \%)$ the assembled swarm-bot failed to overcome the hill. These failures happened when the assembled swarm-bot moved towards the light source with an orientation overly parallel to the orientation of the hill.

Overall, the results show that the additional stability provided by navigating as a larger self-assembled entity (i.e., as a two s-bot swarm-bot instead of individually) caused the task completion rate to increase from $0 \%$ to $35 \%$ (a significant increase according to the two-tailed Fisher's exact test, $p<0.001)$.

\section{EFFICIENCY GAINS THROUGH ADAPTIVITY}

We demonstrate the feasibility of adaptive self-assembly by analysing the behaviour of the adaptive self-assembly controller (see section III-A.1) in different environments, some of which require self-assembly (difficult hill environments), and some of which are navigable individually (moderate hill and no-hill environments). In section IV-A we demonstrate that the system is able to 'choose' appropriate behaviour adaptively based on the environment encountered.

In section IV-B we quantify the benefits of adaptivity by comparing task completion speed of the adaptive selfassembly controller against that of a non-adaptive controller - the preemptive self-assembly controller. The preemptive self-assembly controller is a modified version of the adaptive self-assembly controller in which the start state is Aggregate instead of Solo_Phototaxis. Using this

\footnotetext{
${ }^{1}$ For a more detailed description of this controller refer to [13].

${ }^{2}$ We make the assumption that the task completion rate of a single robot equals the task completion rate of two independently navigating s-bots.
} 


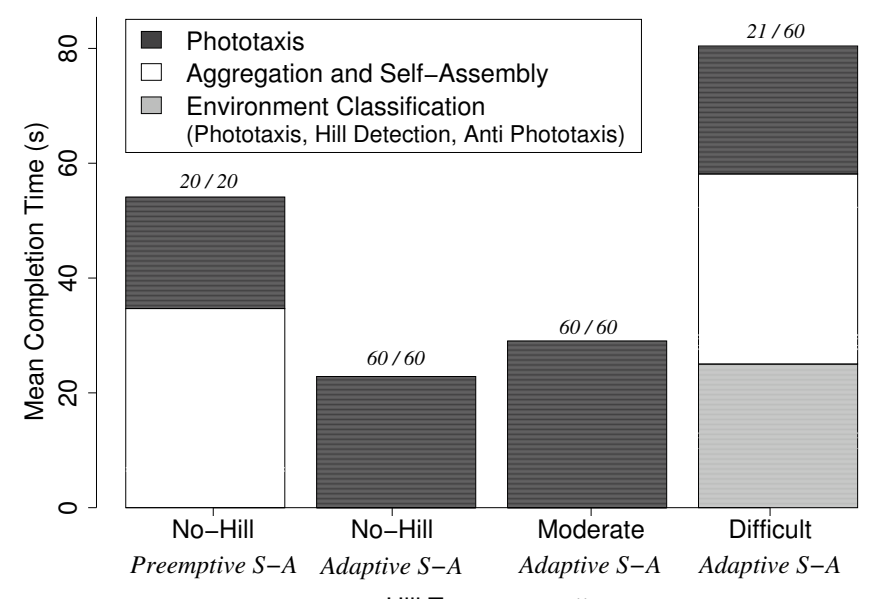

Hill Type; Controller

Fig. 5. Break-down of mean completion times for $s$-bots using the preemptive self-assembly controller (left bar) and the adaptive self-assembly controller (right three bars) in no-hill, moderate hill and difficult hill environments. Only data from completed trials are presented (number of completed trials and number of trials in total are indicated above each bar).

controller, the $s$-bots thus aggregate and self-assemble irrespective of the environment. The connected swarm-bot entity then performs group phototaxis to the light source.

\section{A. Validation of the Adaptive Mechanism}

With the adaptive self-assembly controller, we conducted 60 trials in the difficult hill environments (already discussed in section III-B), 60 trials in the moderate hill environments and 20 trials in the no-hill environment.

In the 60 trials in the difficult hill environments, both $s$ bots successfully detected the slope in every trial and 'chose' to assemble into a two s-bot swarm-bot. In the 80 trials in the moderate hill and no-hill environments, both $s$-bots correctly 'chose' not to self-assemble and to navigate to the target individually. Thus the adaptive mechanism correctly classified the environment in all 140 trials.

\section{B. Comparative Performance}

We conducted 20 trials with the preemptive self-assembly controller in the no-hill environment. This provides a baseline against which we compare the adaptive self-assembly controller. Throughout this section we assume that the mean completion time of the preemptive self-assembly controller in the no-hill environment is a lower bound for the mean completion time of the same controller in moderate hill or difficult hill environments.

Fig. 5 shows a break-down of mean completion times for the preemptive self-assembly controller and the adaptive selfassembly controllers in no-hill, moderate hill and difficult hill environments.

In the no-hill environment, the adaptive self-assembly controller performed significantly better than the preemptive selfassembly controller (two-tailed Mann-Whitney, $p<0.001$ ). The mean task completion times were respectively $22.9 \mathrm{~s}$ and

\footnotetext{
$\dagger$ Upper bound percentage, as it is calculated using a lower bound for the mean completion time of the preemptive self-assembly controller.
}

$54.1 \mathrm{~s}$. The adaptive self-assembly controller took on average $57.7 \%$ less time to complete the task than the preemptive selfassembly controller. Looking at the break-down of the mean completion times in Fig. 5, we can see that $s$-bots using the preemptive self-assembly controller spent over half of their time on actions that were not necessary to complete the task (i.e., aggregation and self-assembly).

In the moderate hill environments, the mean completion time for s-bots using the adaptive self-assembly controller was $29.0 \mathrm{~s}$, which is $27.1 \%$ more than the mean completion time for the same controller in the no-hill environment. This increase is due to the extra overhead of environment classification, which takes place during phototaxis $-s$-bots using the adaptive self-assembly controller slow down on the slope to test its navigability. Nevertheless, even using the lower bound mean completion time for the preemptive selfassembly controller, the adaptive self-assembly controller still significantly outperforms the preemptive self-assembly controller (two-tailed Mann-Whitney, $p<0.001$ ). In the moderate hill environments, the mean completion time for the adaptive self-assembly controller was $46.4 \%$ less than the lower bound mean completion time for the preemptive self-assembly controller.

In contrast, in environments where self-assembly is necessary (difficult hill environments), it is intuitively clear that the preemptive self-assembly controller is more efficient than the adaptive self-assembly controller. S-bots using the adaptive self-assembly controller have a major extra overhead of environment classification consisting of initial solo-phototaxis, hill detection and anti-phototaxis (see Fig. 5). The mean completion time for the adaptive self-assembly controller in the difficult hill environments was $80.4 \mathrm{~s}$.

Thus, the relative frequency with which the s-bots encounter the different environments determines which of the two controllers is more efficient. If we consider a distribution of environments containing only no-hill and difficult hill environments, then we can use the mean completion times to calculate the upper bound percentage of no-hill environments encountered, $\alpha$, for which the efficiency of the two controllers is identical ${ }^{\dagger}$ :

$$
\begin{aligned}
22.9 \alpha+80.4(1-\alpha) & =54.1 \\
\Rightarrow \alpha & =0.457
\end{aligned}
$$

We conclude that the adaptive self-assembly controller is more efficient than the preemptive self-assembly controller if more than $45.7 \%^{\dagger}$ of encountered environments are no-hill environments. If we consider a distribution of environments containing only moderate hill environments and difficult hill environments, a similar analysis reveals that the adaptive self-assembly controller will be more efficient if at least $51.3 \%^{\dagger}$ of the environments are moderate hill environments.

\section{Benefits of Morphology Awareness}

Using the adaptive self-assembly controller, the $s$-bots always correctly responded to the difficult hill environments by self-assembling. However, the overall task completion 

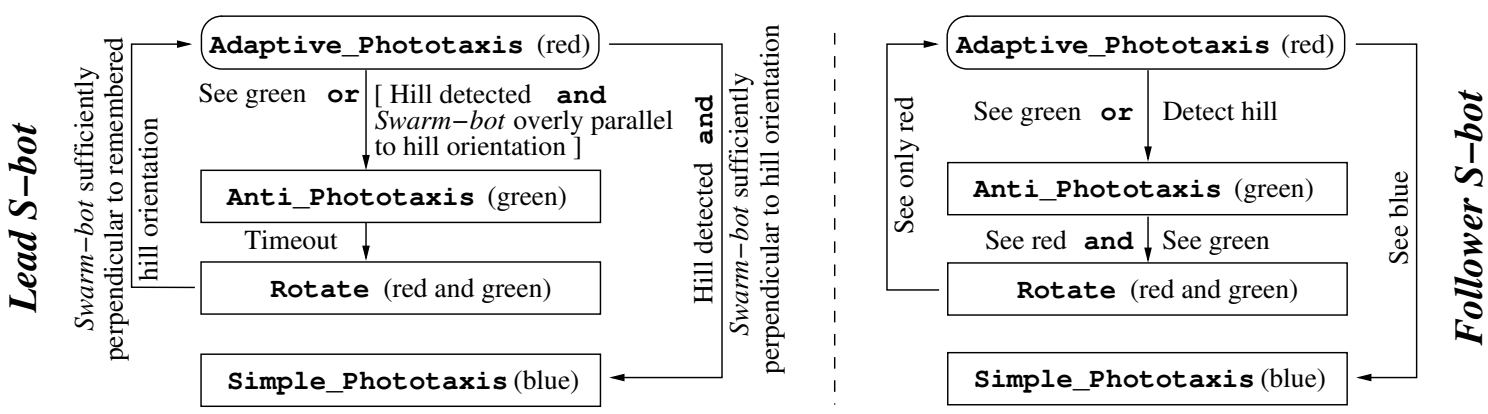

Fig. 6. The morphology aware controller - finite state machine extension of the adaptive self-assembly controller. The s-bot first executes the adaptive self-assembly controller (see Fig. 3). However, instead of executing the Group_Phototaxis state the $s$-bot switches into state Adaptive_Phototaxis, either as a lead s-bot (the s-bot that seeded self-assembly) or as a follower s-bot (all other s-bots).

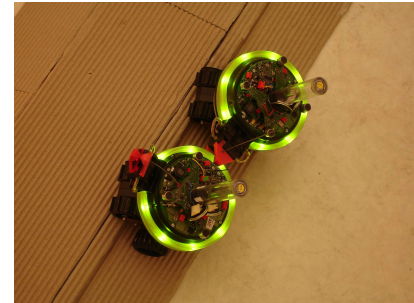

(a)

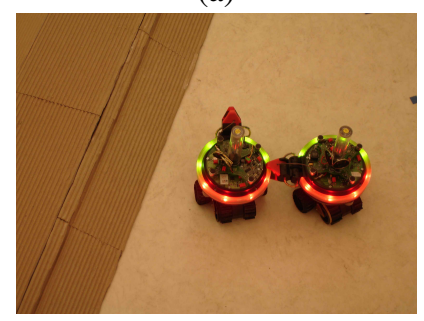

(c)

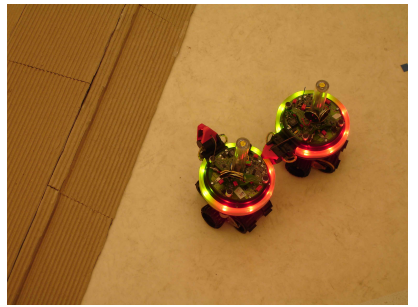

(b)

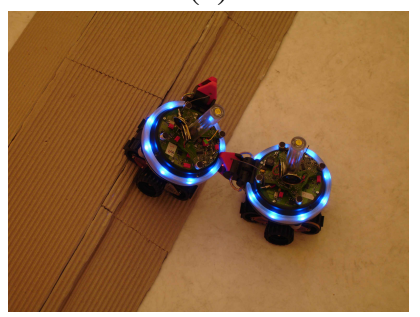

(d)
Fig. 7. Execution of the morphology aware controller. (a): The s-bots have already adaptively aggregated and self-assembled. The connected swarmbot approaches the hill with an inappropriate orientation. (b): The swarmbot performs antiphototaxis away from the hill and starts to rotate. (c): The swarm-bot rotates until it has a more appropriate orientation (based on memory of the hill orientation). (d): The swarm-bot recognises that it has an appropriate orientation and overcomes the hill.

rate for these environments was not high - only $35 \%$ of the s-bots completed the task. This is because a two s-bot swarm-bot topples over whenever it approaches the difficult hill with an orientation that is insufficiently perpendicular to the orientation of the hill. Such inappropriate swarmbot orientations occurred frequently in the difficult hill experiments, as the approach orientation of the swarm-bot was purely random. Immediately after self-assembling into a swarm-bot, the constituent s-bots would both just perform phototaxis. Thus the swarm-bot would approach the hill with whatever orientation it happened to assume as it was formed.

In this section, we present a mechanism which allows the $s$-bots to leverage the morphology of the connected swarmbot in a task oriented way. The s-bots ensure that the swarmbot is appropriately rotated with respect to any rough terrain encountered so as to keep the vertical projection of the swarm-bot's center of gravity inside its footprint.

\section{A. Morphology Aware Controller}

We extended the adaptive self-assembly controller (see Fig. 3) to allow a swarm-bot that assembles with a linear morphology to detect the orientation of a hill and rotate in order to approach the hill at an appropriate angle. ${ }^{3}$

Fig. 6 shows the finite state machine extension for the morphology aware controller. Fig. 7 illustrates a two $s$-bot system executing the morphology aware controller. The s-bots start by executing the adaptive self-assembly controller. However, instead of executing the final Group_Phototaxis state of the adaptive self-assembly controller, the s-bots start execution of the morphology aware controller in state Adaptive_Phototaxis.

In state Adaptive_Phototaxis the s-bots perform collective phototaxis while constantly checking the orientation of any hills they encounter with respect to the orientation of the swarm-bot (each s-bot uses its 3D accelerometers to determine the orientation of the hill and its camera to determine the orientation of the swarm-bot). If a hill is encountered and the swarm-bot is appropriately rotated with respect to the hill (perpendicular to the orientation of the hill with a tolerance of $20^{\circ}$ ) the $s$-bots continue performing phototaxis to the light source, but no longer check the orientation of any encountered hills (state Simple_Phototaxis). If the swarm-bot is not appropriately rotated, the s-bots remember the orientation of the hill and retreat away from the hill for a given length of time (state Anti_Phototaxis). They rotate until the swarm-bot is appropriately rotated with respect to the remembered hill orientation (state Rotate), and then start performing collective phototaxis again (state Adaptive_Phototaxis).

We use a leader-follower architecture. The s-bot that seeded the self-assembly process becomes the lead s-bot and is responsible for determining whether or not the swarm-bot is appropriately rotated. Using its LEDs, the lead s-bot issues

\footnotetext{
${ }^{3}$ Note that in our experiments, since there are only two $s$-bots selfassembling, the assembled swarm-bot must be linear, and there will always be a single lead s-bot and a single follower s-bot. The controller has, however, been written to be applicable to a linear swarm-bot of arbitrary length - hence the instruction propagation mechanism. To let more than two s-bots self-assemble into a linear formation, a more elaborate control of the self-assembly process would be required. This is a subject of ongoing research [3].
} 
instructions to advance, retreat or rotate to all other $s$-bots (follower s-bots) in the swarm-bot. Follower s-bots illuminate their own LEDs to mimic the LEDs of the $s$-bot they are gripping (which is guaranteed to be closer to the lead $s$-bot in a linear morphology). In this way instructions propagate along the swarm-bot from the lead s-bot to all the follower $s$-bots. The only exception is if a follower s-bot detects the hill before the lead s-bot, in which case the instruction to retreat propagates in the other direction.

Below, we detail the different states of the morphology aware controller and the state transition conditions.

\section{State Adaptive_Phototaxis}

Both the lead s-bot and the follower s-bot illuminate their red LEDs.

1) Lead S-Bot: If the lead s-bot detects a hill, it monitors whether or not the swarm-bot is appropriately rotated with respect to the orientation of the hill. Orientation of the hill is measured using the accelerometers. Orientation of the swarm-bot is calculated based on the positions of nearby LEDs detected with the camera. If the swarmbot is appropriately rotated, the lead s-bot switches into state Simple_Phototaxis. Otherwise, the lead s-bot switches into state Anti_Phototaxis. When the lead sbot switches into state Anti_Phototaxis it stores the orientation of the hill with respect to the target light source for later use in state Rotate.

If the lead s-bot perceives green, it assumes that a follower $s$-bot of the linear swarm-bot has detected the presence of the hill, and therefore switches into state Anti_Phototaxis. In this case, it uses the direction towards the nearest follower s-bot (instead of its accelerometers) to estimate the orientation of the hill.

2) Follower S-Bot: If the follower s-bot detects a hill or perceives green, it switches into state Anti_Phototaxis. The follower s-bot does not check the alignment of the swarm-bot.

\section{State Anti_Phototaxis}

Both the lead s-bot and the follower s-bot illuminate their green LEDs. The lead s-bot switches to state Rotate after a timeout. Follower s-bots switch into state Rotate when they see red and green LEDs in front of them.

\section{State Rotate}

3) Lead S-Bot: The lead s-bot continually compares the current orientation of the swarm-bot against the remembered hill orientation. If the swarm-bot is appropriately oriented and the lead s-bot is at the end of the swarmbot closest to the hill, the lead s-bot switches into state Adaptive_Phototaxis. Otherwise, the swarm-bot needs to rotate-the lead s-bot moves in a direction perpendicular to the orientation of the swarm-bot so as to rotate the swarmbot either clockwise or anti-clockwise as appropriate.

While rotating, the lead s-bot communicates rotation instructions to the follower s-bots by illuminating its green
TABLE I

EXPERIMENTAL RESULTS FOR ENVIRONMENTS WITH THE DIFFICULT HILL. EACH CONTROLLER WAS EVALUATED IN 60 INDEPENDENT TRIALS (20 TRIALS PER HILL ORIENTATION).

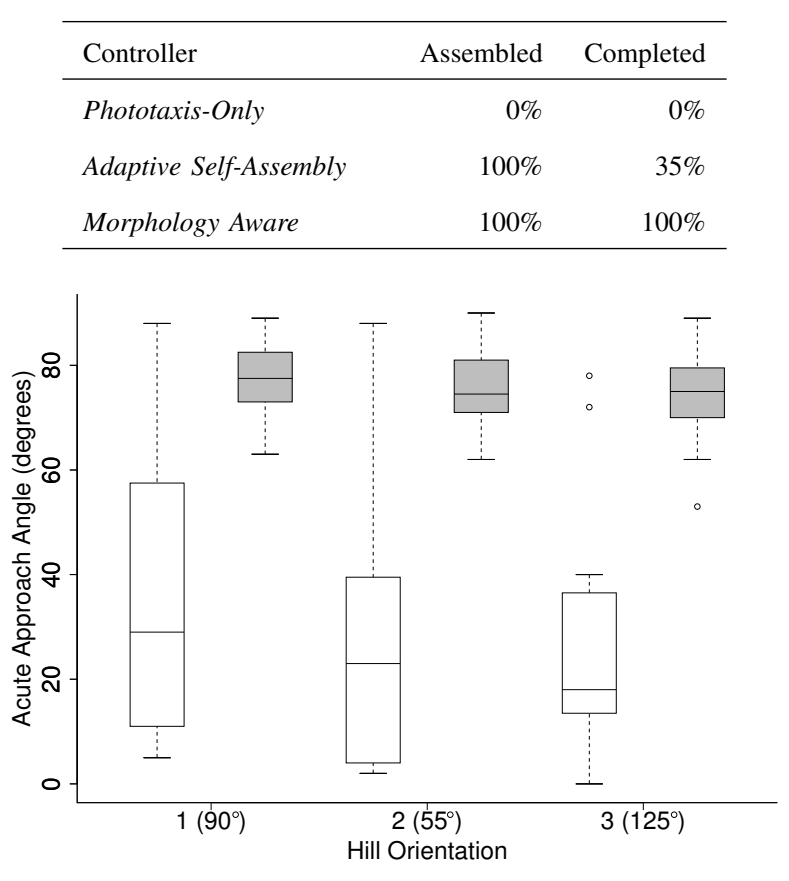

Fig. 8. Box-and-whisker plot showing acute approach angles (orientation of the swarm-bot with respect to the orientation of the hill when the swarm-bot first makes contact with the hill). If the swarm-bot approached the hill more than once, only data from the final approach is shown. Each box represents 20 trials. White boxes: swarm-bots without morphology awareness. Gray boxes: swarm-bots with morphology awareness.

LEDs in the direction in which it is moving and its red LEDs in the opposite direction.

4) Follower S-Bot: If the follower s-bot can see red to its left and green to its right, it interprets this as an instruction to rotate the swarm-bot clockwise. It therefore moves to its left (in Fig. 7 the $s$-bot furthest from the hill is a follower s-bot carrying out a 'rotate clockwise' instruction). If the follower $s$-bot can see red to its right and green to its left, it instead rotates the swarm-bot anti-clockwise by moving to its right. If the follower s-bot only detects red (without any green), it switches into state Adaptive_Phototaxis.

\section{State Simple_Phototaxis}

Both the lead s-bot and the follower s-bot illuminate their blue LEDs and perform collective phototaxis.

\section{B. Results}

Table I summarises the results for the morphology aware controller in the difficult hill environments. Over 60 trials, the robots achieved the optimal task completion rate of $100 \%$ (see last row of the table). This increase in task completion rate can be attributed to morphology awareness, that ensures the swarm-bot is appropriately rotated with respect to the hill. The effectiveness of the rotation mechanism can been seen in Fig. 8. Morphology aware swarm-bots orient themselves 
against each of the three hill orientations significantly better than swarm-bots that are not morphology aware (two-tailed Mann-Whitney, $p<0.001$ ).

When the s-bots leveraged the morphology of the connected swarm-bot in a task specific way, they achieved an optimal completion rate. Note, however, that the morphology aware controller required additional time for task completion, as the swarm-bot has to retreat back from the hill, rotate, and approach the hill with a different orientation. The rotation mechanism on average took up $37.1 \%$ of the total task completion time.

\section{CONCLUSION}

Groups of autonomous robots can use self-assembly to work together and overcome the physical limitations of individual robots. In this study we presented a quantitative analysis of the performance benefits of self-assembly in a robotic system. We showed that simple advantages of scale derived from acting as a physically larger selfassembled entity allowed for a significant improvement in task completion (from $0 \%$ to $35 \%$ ). We went on to show that significant benefits in system efficiency could be derived by making self-assembly adaptive-allowing the robots to choose when and if to self-assemble based on the nature of the environments they encounter. Finally, we demonstrated that significant improvements in task completion (from 35\% to $100 \%$ ) could be achieved if the robots leveraged their connected morphology in a task specific way.

The controllers used in this study were designed to be scalable. In particular, each robot executed the same finite state machine independently and only local (visual) communication was used. The scalability of the core self-assembly component has been verified previously with up to 16 real robots [6]. It remains, however, to test the scalability of the adaptive self-assembly mechanism and of the morphology aware controller. This will require the design of a new robotic task, since the current robotic task has already been solved optimally with two robots.

Our system was designed to leverage a particular connected morphology, but had no way of selecting what morphology was formed. We believe that robotic systems could benefit by using 'morphology selection' in addition to the 'morphology awareness' we demonstrated in this paper. To this end, we are currently investigating ways of generating specific connected morphologies using selfassembling robots [3].

\section{ACKNOWLEDGEMENTS}

This work was supported by the ANTS project, an Action de Recherche Concertée funded by the Scientific Research Directorate of the French Community of Belgium; by the SWARMANOID project, funded by the Future and Emerging Technologies programme of the European Commission (grant IST-022888); and by COMP2SYS, a Marie Curie Early Stage Research Training Site funded by the European Community's Sixth Framework Programme (grant MESTCT-2004-505079). The information provided is the sole responsibility of the authors and does not reflect the Community's opinion. The Community is not responsible for any use that might be made of data in this publication. Roderich Groß acknowledges support by the European Commission through the Marie Curie Transfer of Knowledge project BRIDGET (MKTD-CD 2005029961). Marco Dorigo acknowledges support from the Belgian FNRS, of which he is a Research Director.

\section{REFERENCES}

[1] C. Anderson, G. Theraulaz, and J.-L. Deneubourg. Self-assemblages in insect societies. Insectes Soc., 49(2):99-110, 2002.

[2] D. L. D. Caspar. Design principles in organized biological structures. In G. E. W. Wolstenholme and M. O'Connor, editors, Principles of Biomolecular Organization, pages 7-34. J. \& A. Churchill Ltd., London, 1966.

[3] A. L. Christensen, R. O'Grady, and M. Dorigo. Morphology control in a self-assembling multi-robot system. IEEE Robot. Automat. Mag., (in press), 2007. Special Issue: Robotic Self-Diagnosis, Self-Repair, Self-Replication and Self-Assembly.

[4] T. Fukuda, S. Nakagawa, Y. Kawauchi, and M. Buss. Self organizing robots based on cell structures-CEBOT. In Proc. of the 1988 IEEE Int. Workshop on Intelligent Robots, pages 145-150. IEEE Computer Society Press, Los Alamitos, CA, 1988.

[5] T. Fukuda and T. Ueyama. Cellular Robotics and Micro Robotic Systems. World Scientific Publishing, London, 1994.

[6] R. Groß, M. Bonani, F. Mondada, and M. Dorigo. Autonomous selfassembly in swarm-bots. IEEE Trans. Robot., 22(6):1115-1130, 2006.

[7] S. Hirose, T. Shirasu, and E. F. Fukushima. Proposal for cooperative robot "Gunryu" composed of autonomous segments. Robot. Auton. Syst., 17(1-2):107-118, 1996.

[8] H. Jacobson. On models of reproduction. Am. Sci., 46:255-284, September 1958.

[9] A. Lioni, C. Sauwens, G. Theraulaz, and J.-L. Deneubourg. Chain formation in Ecophylla longinoda. J. Insect Behav., 14(5):679-696, 2001.

[10] F. Mondada, M. Bonani, A. Guignard, S. Magnenat, C. Studer, and D. Floreano. Superlinear physical performances in a SWARM-BOT. In Advances in Artificial Life: 8th European Conf., ECAL 2005, volume 3630 of Lecture Notes in Artificial Intelligence, pages 282291. Springer-Verlag, Berlin, Germany, 2005.

[11] F. Mondada, L.M. Gambardella, D. Floreano, S. Nolfi, J.-L. Deneubourg, and M. Dorigo. The cooperation of swarm-bots: Physical interactions in collective robotics. IEEE Robot. Automat. Mag., 12(2):21-28, 2005.

[12] S. Murata, K. Kakomura, and H. Kurokawa. Docking experiments of a modular robot by visual feedback. In Proc. of the 2006 IEEE/RSJ Int. Conf. on Intelligent Robots and Systems, pages 625-630. IEEE Computer Society Press, Los Alamitos, CA, 2006.

[13] R. O’Grady, R. Groß, F. Mondada, M. Bonani, and M. Dorigo. Selfassembly on demand in a group of physical autonomous mobile robots navigating rough terrain. In Advances in Artificial Life: 8th European Conf., ECAL 2005, volume 3630 of Lecture Notes in Artificial Intelligence, pages 272-281. Springer-Verlag, Berlin, Germany, 2005.

[14] M. Rubenstein, K. Payne, P. Will, and W.-M. Shen. Docking among independent and autonomous CONRO self-reconfigurable robots. In Proc. of the 2004 IEEE Int. Conf. on Robotics and Automation, volume 3, pages 2877-2882. IEEE Computer Society Press, Los Alamitos, CA, 2004.

[15] E. Tuci, R. Groß, V. Trianni, F. Mondada, M. Bonani, and M. Dorigo. Cooperation through self-assembly in multi-robot systems. ACM Trans. Auton. Adapt. Syst., 1(2):115-150, 2006.

[16] G. M. Whitesides and B. Grzybowski. Self-assembly at all scales. Science, 295(5564):2418-2421, 2002.

[17] M. Yim, Y. Zhang, K. Roufas, D. Duff, and C. Eldershaw. Connecting and disconnecting for chain self-reconfiguration with PolyBot. IEEE/ASME Trans. Mechatron., 7(4):442-451, 2002.

[18] V. Zykov, E. Mytilinaios, B. Adams, and H. Lipson. Self-reproducing machines. Nature, 435(7039):163-164, 2005. 